\title{
Room-Temperature Phosphorescence of a Supercooled Liquid: Kinetic Stabilization by Desymmetrization
}

\author{
Mao Komura, Takuji Ogawa, and Yosuke Tani*[a]
}

[a] Mao Komura, Prof. Dr. T. Ogawa, Prof. Dr. Y. Tani

Department of Chemistry

Graduate School of Science, Osaka University

1-1 Machikaneyama, Toyonaka, Osaka 560-0043, Japan

E-mail: y-tani@chem.sci.osaka-u.ac.jp

Supporting information for this article is given via a link at the end of the document

\begin{abstract}
Solvent-free liquid organic phosphors are of significant interest because they are flexible and processable, which makes them ideal for various applications, including organic light-emitting diodes. However, achieving liquid organic room-temperature phosphorescence (RTP) is a challenging task because the liquid state provides a less rigid environment than the crystal. Here, we report that an unsymmetrical heteroaromatic 1,2-diketone forms a kinetically stable supercooled liquid that exhibits RTP and thermochromic phosphorescence. The diketone core is flexible, with eight distinct conformers possible, which prevents nucleation for liquid-solid transition. Notably, RTP is unique to the liquid state and is virtually invisible in solution as well as in the crystalline solid state. Our work demonstrates that flexible unsymmetrical skeletons are promising motifs for the further development of liquid organic phosphors.
\end{abstract}

Room-temperature phosphorescence (RTP) is a unique luminescence phenomenon that is useful for a number of applications, including organic light-emitting diodes (OLEDs), bio-imaging, and encryption inks. ${ }^{[1]}$ In particular, metal-free organic RTP materials are desirable owing to their cost-effectiveness and low environmental burden. ${ }^{[2]}$ Most organic RTP luminophores require rigid crystal lattices to suppress non-radiative decay that quenches RTP. ${ }^{[3]}$ Since the optical properties of crystalline materials deteriorate through lattice defects, organic RTP materials that function in non-rigid and flexible molecular environments are desired. ${ }^{[4]}$

The liquid state is the most flexible of condensed materials. Owing to their flexibilities and uniformities, liquid chromophores are advantageous because they are easily processed and have defectindependent material properties. ${ }^{[5]}$ Achieving solvent-free liquid RTP not only simplifies OLED device structure and fabrication processes but is also useful for flexible and wearable light-emitting devices. ${ }^{\left[{ }^{[5}\right.}$ Moreover, solvent-free liquid materials can exploit high chromophore concentrations, unlike solution- or polymer-based materials. However RTP is hardly observed in the liquid state, where molecules move more freely than in the solid state. To the best of our knowledge, only a pioneering two-component liquid system has been reported to show practical organic RTP. ${ }^{[6]}$ Babu et al. demonstrated that the introduction of a long, branched alkyl chain onto a rigid bromonaphthalimide chromophore produces a solvent-free liquid that exhibits visible RTP when mixed with carbonyl compounds; however, the liquid itself showed virtually no RTP as a single component (Figure 1a).

We previously found that thienyl diketone derivatives exhibit more efficient RTP in the amorphous solid states than in the crystal, with phosphorescence quantum yield $\Phi_{\mathrm{p}}$ of up to $10 \%$, unaided by any metal heavy atom effect (Figure 1b). ${ }^{[7]}$ Notably, unlike typical chromophores with rigid skeletons, the diketone skeleton is flexible with five distinct conformers. Among them, a trans-planar (TP) conformer has two-fold intramolecular chalcogen bonds and is responsible for the efficient RTP in the amorphous solid state despite its minor proportion. We also demonstrated that desymmetrizing $C_{2}$ symmetrical diketone 1-by replacing one bromine with hydrogenreduced intermolecular interactions in the crystals, dramatically lowered the melting point, and made the crystal non-emissive while maintaining the emissivity of the amorphous solid (Figure 1b). ${ }^{[7 b]}$ Consequently, the unsymmetrical diketone crystal showed, for the first time, turn-on RTP in response to mechanical stimuli in a metal-free organic molecule. These findings prompted us to expand the utility of the desymmetrization approach. ${ }^{[8]}$

a) Rigid Core with Long Alkyl Chains (Babu et al., 2019)

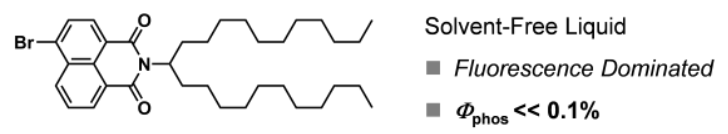

b) Desymmetrization of a Thienyl Diketone

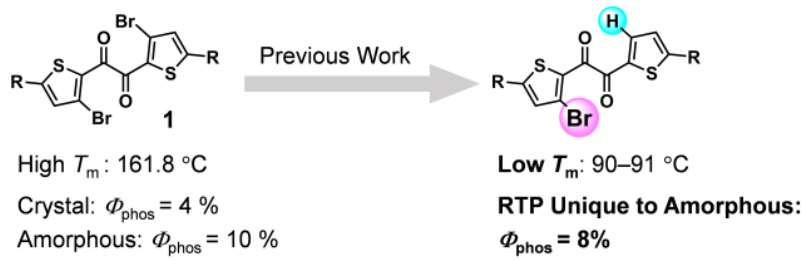

1

c) This Work: Desymmetrization Approach for Liquid RTP

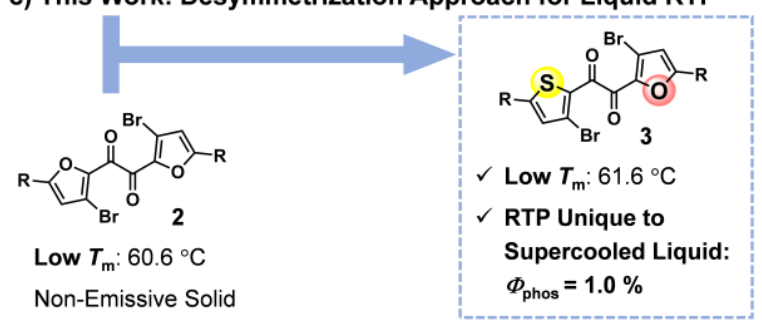

Figure 1. Strategy toward solvent-free liquid organic RTP. a) Introducing long alkyl chains into a rigid bromonaphthalimide chromophore. b, c) Desymmetrizing $C_{2}$-symmetrical thienyl diketones. $\mathrm{R}=$ triisopropylsilyl, $T_{\mathrm{m}}$ : melting point. 
In this work, we envisaged that desymmetrization provides a practical approach to achieving solvent-free liquid organic RTP (Figure 1c). Unsymmetrical diketone $\mathbf{3}$ was designed to combine the characteristics of two $C_{2}$-symmetrical diketones, namely the efficient RTP of $\mathbf{1}$ and the low melting point of furyl diketone 2. Herein, we report RTP in a solvent-free liquid state of the heteroaromatic 1,2diketone 3. Moreover, liquid 3 exhibits temperature-dependent phosphorescent color (i.e., thermochromism).

Diketone $\mathbf{3}$ was synthesized by the cross-benzoin condensation of the corresponding aldehydes, followed by oxidation (see Supporting Information (SI) for details). ${ }^{[7]}$ After purification, overnight solvent removal at reduced pressure and $40{ }^{\circ} \mathrm{C}$ afforded 3 as a liquid; asprepared 3 was confirmed to be solvent-free by ${ }^{1} \mathrm{H}$ NMR and ${ }^{13} \mathrm{C}$ NMR spectroscopy, and elemental analysis. Liquid 3 is isotropic, as confirmed by X-ray diffractometry (XRD) and visual inspection under a polarizing optical microscope (Figures S1 and S2).

In air at room temperature, solvent-free liquid $\mathbf{3}$ exhibited orange phosphorescence without discernible fluorescence; its steady-state photoluminescence $(\mathrm{PL})$ spectrum exhibits an emission maximum at $574 \mathrm{~nm}$ with a PL lifetime of $16 \mu \mathrm{s}$, consistent with phosphorescent emission (Figure 2). The $\phi_{p}$ value was found to be $1.0 \%$, which is the highest among single-component organic liquids. ${ }^{[6]}$ We suggest that the RTP of liquid $\mathbf{3}$ originates from the TP conformer (Figure 3). The $\mathrm{PL}$ spectrum of liquid $\mathbf{3}$ is quite similar to that of $\mathbf{1}$ in solution, whose RTP originates from the corresponding TP conformer (Figure S4). ${ }^{[7]}$ Density functional theory (DFT) calculations at the UB3LYP-D3/6$311 \mathrm{G}(\mathrm{d})$ level of theory suggested that the most stable conformer of 3' (in which the silyl groups in $\mathbf{3}$ are replaced by $\mathrm{H}$ ) in the lowest triplet state $\left(T_{1}\right)$ is the TP conformer (Figures 3 and $S 9$, and Table $S 1$ ). Timedependent (TD)-DFT calculations revealed that the $T_{1}-S_{0}$ radiative transition (phosphorescence) principally consists of the HOMO-LUMO transition (94\%) with an electronic configuration with ${ }^{3}\left(n, \pi^{\star}\right)$ character, which is favorable for spin inversion according to ElSayed's rule. ${ }^{[1]}$ Moreover, bromine atoms close to the carbonyl oxygen further promote phosphorescence decay by an intramolecularly directed heavy atom effect. ${ }^{[9]}$
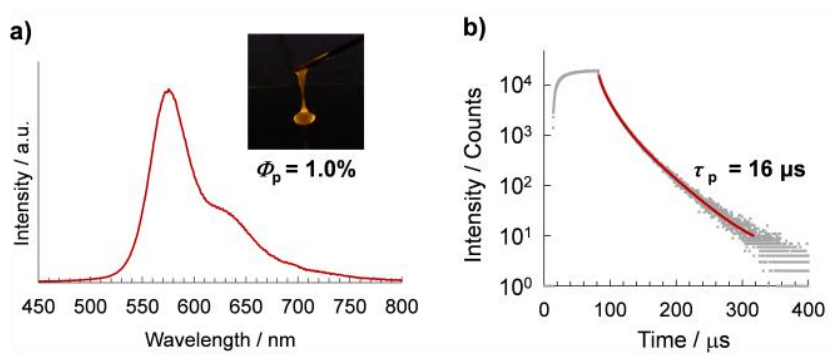

Figure 2. (a) Steady-state photoluminescence spectrum and (b) decay profile recorded at $570 \mathrm{~nm}$ of liquid 3 in air at room temperature $\left(\lambda_{\mathrm{ex}}=368 \mathrm{~nm}\right)$. Inset: photographic image of liquid 3 when irradiated with UV light $(365 \mathrm{~nm})$. Absolute $\Phi_{\mathrm{p}}$ was determined using an integrating sphere device.

The RTP of $\mathbf{3}$ was only visible in its solvent-free liquid state. In solution, 3 was virtually nonemissive, even under $\operatorname{Ar}\left(\phi_{p}=0.04 \%, 2.2\right.$ $\times 10^{-4} \mathrm{M}$ in cyclohexane). The PL spectrum of solution-phase 3 is in good agreement with that of the solvent-free liquid, which indicates that the emissive conformers are the same for both states (Figure S4). However, the phosphorescence decay rate constants $\left(k_{\mathrm{p}}\right)$, which reflect molecular electronic properties, are significantly different (Table 1). ${ }^{[10]}$ The liquid exhibits a $k_{p}$ that is nearly 50 -times larger than the solution, which implies that the external heavy atom effect from adjacent molecules contributes in the condensed solvent-free liquid. ${ }^{[1]}$ In contrast, the non-radiative decay rate constants $\left(k_{n r}\right)$ of the solventfree liquid in air and the degassed solution are not significantly different (Table 1). The RTP intensity of the liquid is only 1.47-times higher under $\mathrm{N}_{2}$, suggesting that the liquid is sufficiently viscus to retard oxygen diffusion, thereby suppressing phosphorescence quenching (Figure S3). Nonetheless, we emphasize that the RTP enhancement of liquid $\mathbf{3}$ originates not from the decrease in $k_{n r}$, but from the increase in $k_{\mathrm{p}}$.

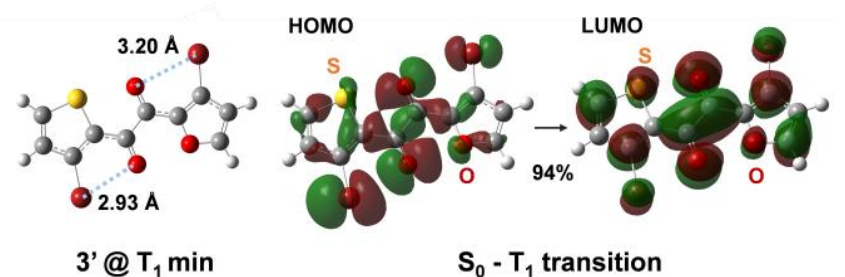

Figure 3. Geometry of $\mathrm{T}_{1}$-minimum-energy TP conformer of 3' and its KohnSham HOMO and LUMO orbitals, calculated at the (TD-)UB3LYP-D3/6-311G(d) level of theory.

Table 1: Photophysical properties of $\mathbf{3}$ at room temperature.

\begin{tabular}{ccccc}
\hline & $\Phi_{\mathrm{p}}$ & $\tau_{\mathrm{p}} / \mu \mathrm{s}$ & $k_{\mathrm{p}} / \mathrm{s}^{-1[\mathrm{a}]}$ & $k_{\mathrm{nr}} / \mathrm{s}^{-1[\mathrm{a}]}$ \\
\hline Solvent-free liquid $^{[\mathrm{b}]}$ & $1.0 \%[\mathrm{c}]$ & 16 & 650 & 62000 \\
Cyclohexane solution $^{[\mathrm{d}]}$ & $0.04 \%{ }^{[\mathrm{e}]}$ & 29 & 14 & 34000 \\
\hline
\end{tabular}

[a] Calculated according to the formulas: $k_{\mathrm{p}}=\Phi_{\mathrm{p}} / \tau_{\mathrm{p}}$ and $k_{\mathrm{nr}}=\left(1-\Phi_{\mathrm{p}}\right) / \tau_{\mathrm{p}}$. [b] In air. [c] Absolute $\Phi_{p}$ determined using an integrating sphere device. [d] Under Ar. [e] Relative $\Phi_{p}$ determined using quinine sulfate as the standard. ${ }^{[11]}$

Diketone 3 remained liquid at room temperature for at least three months and eventually solidified and became nonemissive (Figure S10). The solid was subjected to XRD, which showed sharp diffraction peaks that demonstrate that the solid is crystalline with an ordered molecular arrangement (Figure S11). Thus, the RTP of $\mathbf{3}$ is efficient only in the kinetically stable supercooled liquid (SCL) and is invisible either in solution or in the crystalline state. The liquefaction-induced RTP highlights the uniqueness of $\mathbf{3}$, considering that common organic RTP materials require rigid environments. ${ }^{[1-4]}$ We speculated that a disadvantageous skewed conformation and insufficient intermolecular interactions are responsible for the lack of emissivity of solid $\mathbf{3}$, despite its crystallinity, as observed for our previous unsymmetrical diketone (Figure S12). ${ }^{7 b]}$

To gain insight into molecular designs that lead to liquefaction, we compared the stability of the liquid of the unsymmetrical diketone 3 with those of symmetrical diketones $\mathbf{1}$ and $\mathbf{2}$; the melting points of $\mathbf{1}$, 2 , and $\mathbf{3}$ are $161.8,60.0$, and $61.6{ }^{\circ} \mathrm{C}$, respectively, which indicates that the thermodynamic liquid-phase stability of $\mathbf{3}$ is similar to that of 2. In view of kinetic stability (i.e., ease of crystallization from the $\mathrm{SCL}),{ }^{[12]} \mathbf{1}$ instantaneously crystalizes upon evaporation of the solvent, while SCL 2 tends to solidify within a few days at room temperature. Thus, the SCL states of symmetrical diketones are less stable than those of unsymmetrical diketone $\mathbf{3}$ (lasts for more than three months), which implies that kinetic stability is enhanced by desymmetrization. It should be noted that desymmetrization increases the number of (meta-) stable conformers within a $2 \mathrm{kcal} \mathrm{mol}^{-1}$ energy range, from five for the symmetrical core of $\mathbf{1}$ to eight for the unsymmetrical core 
of 3 , as confirmed by DFT calculations (Figures 4, S8, and S9). Moreover, the lack of $C_{2}$ symmetry provides an additional degree of freedom in relative molecular orientation. These features prevent nucleation and growth that form ordered arrangements of crystals, which enhances the kinetic stability of the liquid state.

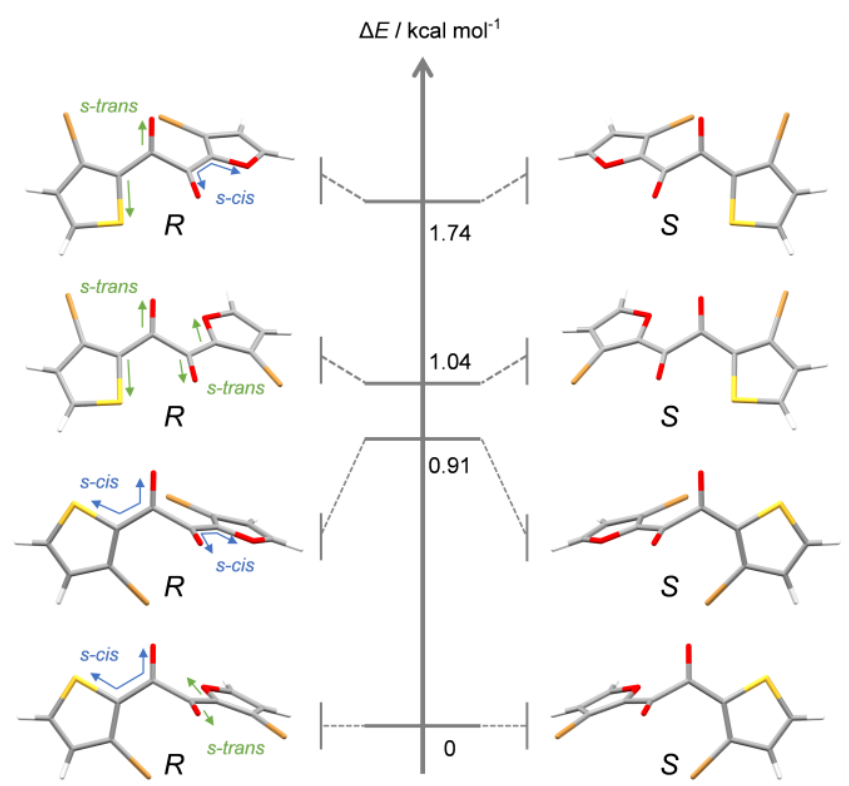

Figure 4. Optimized structures and energy levels of 3' in the $S_{0}$ state calculated at the B3LYP-D3/6-311G(d) level of theory.

With a flexible skeleton liquid phosphor in hand, we next investigated the dependence of phosphorescence on temperature (Figure 5), ${ }^{[13]}$ which revealed that liquid $\mathbf{3}$ is the first metal-free organic molecule to exhibit thermochromic phosphorescence. Steady-state $\mathrm{PL}$ spectra were acquired from -120 to $20^{\circ} \mathrm{C}$ in $10^{\circ} \mathrm{C}$ steps, which showed redshifts in the maximum emission wavelength $\left(\lambda_{\max }\right)$, from $566 \mathrm{~nm}$ to $574 \mathrm{~nm}$. The relationship between $\lambda_{\max }$ and reciprocal temperature can be fitted with two lines, with the slope changing between -10 and $-30{ }^{\circ} \mathrm{C}$. Differential scanning calorimetry (DSC) revealed that 3 has a glass-to-isotropic liquid-transition temperature $\left(T_{\mathrm{g}}\right)$ of $-9.4^{\circ} \mathrm{C}$ (Figure $5 \mathrm{c}$ ), which is close to where the two lines in Figure $5 \mathrm{~b}$ intersect. Therefore, the phosphorescence wavelength of liquid 3 responds to a bulk physical property that discontinuously changes at $T_{\mathrm{g}}$, such as viscosity. ${ }^{[14]}$ We conclude that the increase in viscosity prevents sorrounding molecules from reorganizing as the dipole of the excited molecule changes, which causes liquid $\mathbf{3}$ to exhibit thermochromic behavior (Figure S15).

In summary, efficient RTP in a solvent-free liquid state in air was realized through the desymmetrization of a $C_{2}$-symmetrical thienyl diketone. The unsymmetrical flexible core kinetically stabilizes the SCL state for at least three months. Notably, only the liquid exhibits RTP; the solution and crystalline solid are virtually nonemissive. We suggest that the liquid is sufficiently dense to benefit from the intermolecular (external) heavy atom effect and suppress oxygen diffusion, while being sufficiently flexible to generate an intrinsically emissive TP conformer. In addition, the unsymmetrical diketone exhibits thermochromic phosphorescence, which stems from the fluidity of itself. Our work demonstrates the significance of an unsymmetrical flexible core for realizing RTP in a condensed, yet loose liquid state. Investigation into using the SCL solid-phase transition to develop stimuli-responsive RTP is currently ongoing in our laboratory, along with the development of RTP-emissive thermodynamically stable liquid luminophores.
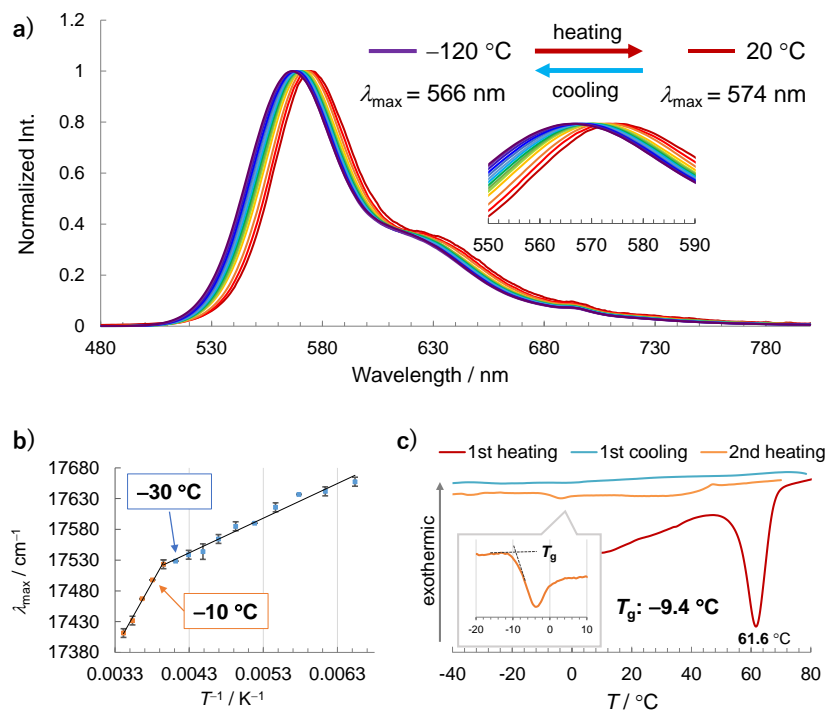

c) -1 st heating -1 st cooling -2 nd heating

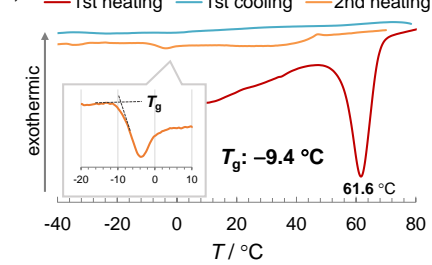

Figure 5. Thermochromic behavior of liquid 3. a) Steady-state PL spectra acquired from -120 to $20^{\circ} \mathrm{C}$ in $10^{\circ} \mathrm{C}$ steps. b) Relationship between $\lambda_{\max }$ and reciplocal temperature. c) DSC thermograms of solid 3 during heating/cooling cycles at $10^{\circ} \mathrm{C} \min ^{-1}$ under a flow of $\mathrm{N}_{2}$.

\section{Acknowledgements}

This work was supported by JSPS KAKENHI (grant number JP19K15542). Y.T. is grateful to the Chubei Itoh Foundation, the ENEOS Tonengeneral Research/Development Encouragement \& Scholarship Foundation, and the Izumi Science and Technology Foundation for financial support. Theoretical calculations were performed at the Research Center for Computational Science, Okazaki, Japan. Some of experiments were performed at the Analytical Instrument Facility, Graduate School of Science, Osaka University.

Keywords: liquids $•$ luminescence $\cdot$ organic room temperature phosphorescence $\cdot$ phase transitions $\bullet$ thermochromism

[1] a) N. J. Turro, Modern Molecular Photochemistry, University Science Books, Sausalito, CA, 1991; b) S. K. Lower, M. A. El-Sayed, Chem. Rev. 1966, 66, 199-241; c) S. Hirata, Adv. Opt. Mater. 2017, 5, 1700116.

[2] a) Kenry, C. Chen, and B. Liu Nat. Commun. 2019, 10, 2111; b) A. D. Nidhankar, Goudappagouda, V. C. Wakchaure, S. S. Babu, Chem. Sci. 2021, 12, 4216-4236.

[3] a) D. B. Clapp, J. Am. Chem. Soc. 1939, 61, 523-524; b) C. S. Bilen, N. Harrison, D. J. Morantz, Nature 1978, 271, 235-237; c) W. Z. Yuan, X. Y. Shen, H. Zhao, J. W. Y. Lam, L. Tang, P. Lu, C. Wang, Y. Liu, Z. Wang, Q. Zheng, J. Z. Sun, Y. Ma, B. Z. Tang, J. Phys. Chem. C 2010, 114, 6090-6099; d) M. Baroncini, G. Bergamini, P. Ceroni, Chem. Commun. 2017, 53, 2081-2093; e) A. Forni, E. Lucenti, C. Botta, E. Cariati, J. Mater. Chem. C, 2018, 6, 4603-4626.

[4] a) W. Zhao, Z. He, B. Z. Tang, Nat. Rev. Mater. 2020, 5, 869-885; b) X. Ma, J. Wang, H. Tian, Acc. Chem. Res. 2019, 52, 738-748; c) N. Gan, H. Shi, Z. An, W. Huang, Adv. Funct. Mater. 2018, 28, 1802657.

[5] a) T. Nakanishi, Functional Organic Liquids, John Wiley \& Sons, Ltd, 2019; b) F. Lu, T. Nakanishi, Adv. Opt. Mater. 2019, 7, 1900176; c) A. 
Ghosh, T. Nakanishi, Chem. Commun. 2017, 53, 10344-10357; d) K. Kushwaha, L. Yu, K. Stranius, S. K. Singh, S. Hultmark, M. N. Iqbal, L. Eriksson, E. Johnston, P. Erhart, C. Müller, K. Börjesson, Adv. Sci. 2019 6, 1801650.

[6] Goudappagouda, A. Manthanath, V. C. Wakchaure, K. C. Ranjeesh, T. Das, K. Vanka, T. Nakanishi, S. S. Babu, Angew. Chem., Int. Ed. 2019 58, 2284-2288; Angew. Chem. 2019, 131, 2306-2310.

[7] a) Y. Tani, M. Terasaki, M. Komura, T. Ogawa, J. Mater. Chem. C 2019 7, 11926-11931; b) Y. Tani, M. Komura, T. Ogawa, Chem. Commun. 2020, 56, 6810-6813.

[8] a) R. J. C. Brown, R. F. C. Brown, J. Chem. Educ. 2000, 77, 724; b) A Gavezzotti, J. Chem. Soc., Perkin Trans. 2 1995, 1399-1404.

[9] O. Bolton, K. Lee, H.-J. Kim, K. Y. Lin, J. Kim, Nat. Chem. 2011, 3, 205 210.

[10] In principle, $\Phi_{\mathrm{p}}$ can be expressed as follows: $\Phi_{\mathrm{p}}=\Phi_{\text {ISc }} k_{\mathrm{p}} /\left(\mathrm{k}_{\mathrm{p}}+\mathrm{k}_{\mathrm{nr}}\right)=\Phi_{\text {ISC }}$ $k_{\mathrm{p}} \tau_{\mathrm{p}}$, where $\Phi_{\text {ssc }}$ is the quantum efficiency of intersystem crossing (ISC). We assumed $\Phi_{\text {ISc }}$ to be unity because no discernible fluorescence was observed. However, the experimentally determined $\Phi_{\mathrm{p}} / \tau_{\mathrm{p}}$ gives $\Phi_{\mathrm{Isc}} k_{\mathrm{p}}$. The larger $\Phi_{\mathrm{p}} / \tau_{\mathrm{p}}$ for the liquid likely indicates a larger $k_{\mathrm{p}}$, but might also indicate a larger $\Phi_{\text {ssc }}$, which is explained by the external heavy atom effects that facilitate the ISC in the liquid.

[11] K. Suzuki, A. Kobayashi, S. Kaneko, K. Takehira, T. Yoshihara, H. Ishida, Y. Shiina, S. Oishi, S. Tobita, Phys. Chem. Chem. Phys. 2009, 11, 98509860.

[12] F. Lu, K. Jang, I. Osica, K. Hagiwara, M. Yoshizawa, M. Ishii, Y. Chino, K. Ohta, K. Ludwichowska, K. J. Kurzydłowski, S. Ishihara, T. Nakanishi, Chem. Sci. 2018, 9, 6774-6778.

[13] a) T. Ogawa, W. M. C. Sameera, M. Yoshida, A. Kobayashi, M. Kato, Dalton Trans. 2018, 47, 5589-5594; b) T. Ogawa, M. Yoshida, H. Ohara, A. Kobayashi, M. Kato, Chem. Commun. 2015, 51, 13377-13380.

[14] M. Han, Y. Tian, Z. Yuan, L. Zhu, B. Ma, Angew. Chem. Int. Ed. 2014, 53, 10908-10912; Angew. Chem. 2014, 126, 11088-11092. 


\section{Entry for the Table of Contents}

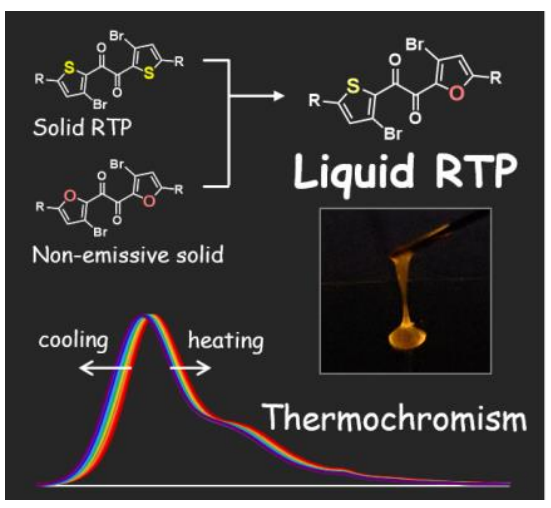

Metal-free, single component liquid room-temperature phosphorescence (RTP) and phosphorescent thermochromism are achieved in air by desymmetrizing a $C_{2}$-symmetrical diketone. The unsymmetrical flexible core with as many as eight conformers kinetically stabilizes the supercooled liquid state. RTP is exclusive to the liquid, which benefits from the external heavy atom effect and suppresses oxygen diffusion, while producing an emissive conformer. 